incorrect. There follows a section on plane waves according to various definitions and a chapter on the asymptotic properties of gravitational fields, Bondi mass loss, "peeling" and so on. Here there are several omissions-Penrose's conformal approach to the peeling theorems, the conserved (?) quantities of Newman and Penrose, definitions of mass and momentum and its relation to the BMS group which should really be included. Work on spinors is alluded to but not described and some NewmanPenrose quantities are incorrectly defined. The last purely theoretical chapter is devoted to the work of Zelmanov.

In his concluding remarks the author states that both from the point of view of experiment and theory much has to be done. With this one can only concur but one cannot help feeling that he is being a little pessimistic in his view of the "problem" of gravitational waves and a little misguided in his search for a unique criterion for gravitation waves - for example, a unique Petrov type. To demonstrate the existence of any kind of radiation it is surely only necessary to show that the physical field in question can transmit energy-momentum and information with a certain speed and without the necessity of a receiver (that is, not inductively). It was the achievement of Bondi and his group first to demonstrate this theoretically in a rigorous fashion; it is to be hoped that the experimental verifications will not be too long coming.

In conclusion then this is an admirable review, with a splendid bibliography, of some aspects of gravitational radiation theory. It is not for the astrophysicists seeking to find ideas for astronomical application or the practical man building apparatus but it should be well suited to people entering the field of mathematically rigorous gravitational radiation theory provided they supplement it with other reading. It would also help them to know that western relativists call "isotropic" "null".

G. W. GibBons

\section{Environmental Poisons}

Environmental Quality and Safety. Edited by F. Coulston and F. Korte. Volume 1. Global Aspects of Chemistry, Toxicology and Technology, as Applied to the Environment. Pp. $\mathrm{x}+267$. (Georg Thieme: Stuttgart; Academic: New York, July 1972.) \$18.

CONCERN over environmental quality and the safety of chemicals released into our environment has been increasing steadily over the past few years. This is also reflected in the phenomenal growth in the literature on environmental pollution and other aspects. Any reasonable attempt at throwing some light on to the confused

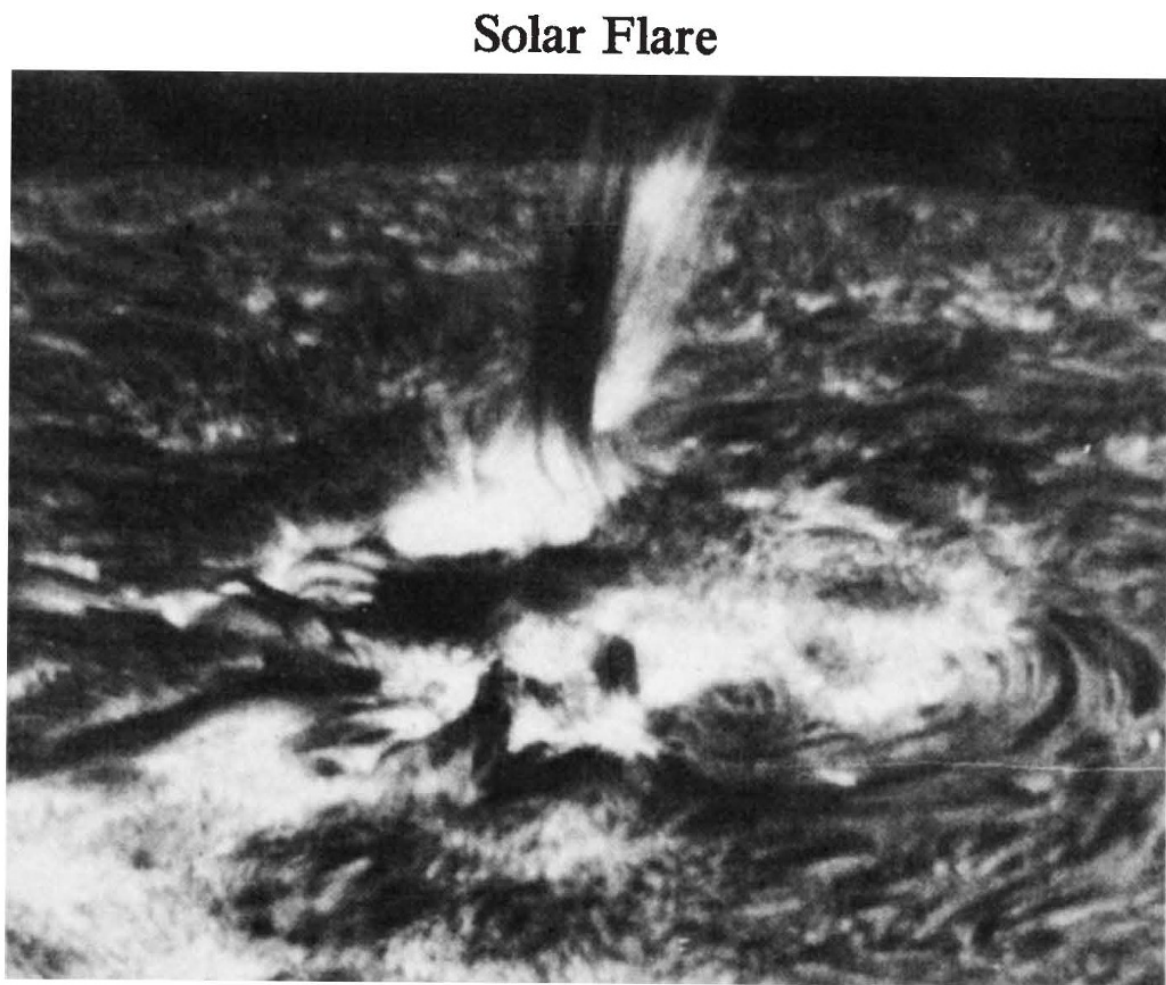

Photograph of active region of the Sun taken in $\mathrm{H \alpha}$ light. Material is shown being ejected close to the limb, photographed on May 22, 1972. From The Quiet Sun, by Edward G. Gibson (National Aeronautics and Space Administration: Washington, 1973, \$6.20).

scene of conflicting opinions presently existing in this field should, therefore, be given full support. Volume 1 of the new series on environmental quality and safety is a brave start if the declared intentions of the editors come to fruition. Perhaps by chance, most of the articles in the first volume are devoted to the toxicology of pesticides. Only one paper by Hasserodt concerns itself with the pollution problem created by man's needs for an ever increasing supply of energy to satisfy the demands of industry and to provide for his comforts. Two out of a total of twenty articles relate to pharmaceutical chemicals. They do not touch on the environmental aspects but are more concerned with standard safety assessments of drugs and profitability considerations.

Pesticides, their environmental behaviour and the implications of their use in agriculture as well as of their residues in food for man as the ultimate consumer, and for the environment generally, are covered in a competent manner. The articles by Matsumura, Brooks and Klein present clear and reasonably comprehensive reviews of the present knowledge of the metabolism of pesticides in microorganisms, insects and plants. However, some statements are not in accordance with presently accepted opinion. Thus on page 60 , the assertion by the author, that $50 \%$ of the lead ingested in food is absorbed, is patently at variance with the generally accepted figure of $10 \%$. Furthermore, on page 61 , to say that the major source of lead in our environment is derived from lead alkyls used in gasoline is incorrect. By far the largest amounts derive from natural geological sources and the enormous industrial usage of lead and its compounds.

How to handle the detailed information on pesticides and arrive at a sensible and satisfactory evaluation of the balance between the benefits and risks of pesticide usage is clearly explained in an excellent article by van Tiel. The ultimate hazard to man from a pesticide depends on his actual exposure to it and all possible metabolic transformation products, if these persist in human food as consumed. The important point is made that only if accurate analytical data are available, as well as adequate determinations of the range of dietary intakes for foodstuffs liable to contain pesticides residues, can the toxicological information be used to make valid and sensible assessments of the health hazards involved.

A reasonable beginning has been made towards more purposeful discussions of environmental hazards and their assessment, but judgment on the real value of this series has to be postponed until further volumes have appeared. Otherwise the book is well produced, the index is not extensive but is adequate and there are only a few printers' errors to mar the generally satisfactory impression. P. S. ElIAS 